\title{
Traer estativo en español de México: su estructura sintáctica y propiedades semánticas*
}

\author{
ÁLVARO RÁBAGO TÁNORI" \\ rabago@uabc.edu.mx \\ LUISA JOSEFINA ALARCÓN NEVE"* \\ alarconlj@yahoo.es
}

Recepción: 24 de octubre de 2014 Aprobación: 02 de marzo de 2015

Forma de citar este artículo: Rábago Tánori, Á., \& Alarcón Neve, L. (2015). Traer estativo en español de México: su estructura sintáctica y propiedades semánticas. Cuadernos de Lingüística Hispánica, 26, 23-47, Tunja: Uptc.

* Artículo de Investigación. El presente trabajo forma parte del proyecto de investigación "Esquemas Sintáctico-Semánticos de traer y llevar en uso estativo-posesivo", aprobado en Consejo Académico y registrado ante el Departamento de Investigación y Posgrado de la Facultad de Lenguas y Letras de la Universidad Autónoma de Querétaro, UAQ.

** Profesor investigador en la Facultad de Idiomas, Mexicali, de la Universidad Autónoma de Baja California, México. Alumno del programa Doctorado en Lingüística, en la Universidad Autónoma de Querétaro. Santiago de Querétaro, México.

*** Doctora en Filología Española. Licenciada en Lengua y Literatura Hispánicas por la Universidad Nacional Autónoma de México, UNAM, y Maestra en Psicología Educativa por la Universidad Autónoma de Querétaro, UAQ. Docente de la Facultad de Lenguas y Letras de la UAQ. Coordinadora del Doctorado en Linguística y Secretaria Académica de dicha facultad. Miembro del Sistema Nacional de Investigadores (SNI) desde 2011. 


\title{
Resumen
}

Esta investigación tuvo como objetivo describir los usos del verbo 'traer' en eventos imperfectivos en el español de México, con el apoyo en la Gramática de Construcciones de Goldberg (1995) y en un estudio previo sobre el 'traer' estativo, de Rábago y Alarcón, 2012. Se exploran algunas aristas sintácticas, semánticas y pragmáticas acerca de la relación entre los participantes: Sujeto, Objeto y sus Complementos. Se analizan ejemplos de dos córpora de registro oral: El Corpus Sociolinguístico de la Ciudad de México y El Corpus del Habla de Monterrey. Se muestra la siguiente tendencia: los objetos alienables aparecen con complementos predicativos, no así con objetos inalienables, donde la estructura preferida se combina con complementos adverbiales o predicados secundarios que habilitan y, en algunos casos, refuerzan la situación discursiva.

Palabras clave: traer estativo, evento imperfectivo, complemento predicativo, complemento adverbial, predicados secundarios, alienable-inalienable, español de México.

\section{The stative verb "Traer" in Mexican Spanish: syntactic structure and semantic properties}

\begin{abstract}
This research project is aimed at describing the uses of verb "traer" in imperfective events in Mexican Spanish, based on the Gramática de Construcciones de Goldberg (1995) and a previous study by Rábago and Alarcón (2012) regarding the stative "traer". A series of syntactic, semantic and pragmatic aspects is explored, regarding the relationships among participants: Subject, Object and their Complements. Examples from two oral register corpora are analyzed: the Sociolinguistic Corpus of Ciudad de México, and the Speech Corpus of Monterrey. The following tendency is shown: alienable objects are used with predicative complements, while inalienable objects combine the preferred structure with adverbial complements or secondary predicates which enable and sometimes strengthen the discursive setting.

Key words: stative verb "traer", imperfective event, predicative complement, adverbial complement, secondary predicate, alienable - inalienable, Mexican Spanish.
\end{abstract}




\section{Apporter étatif en espagnol de Mexico: son structure syntaxique et propriétés sémantiques}

\section{Résumé}

Cette recherche a eu comme objectif la description des usages du verbe "apporter" dans des événements imperfectifs dans l'espagnol de Mexico, ayant comme support la Grammaire de Constructions de Goldberg (1995) et une étude préalable sur le 'apporter' étatif, de Rábago et Alarcón, 2012. On explore quelques intersections syntaxiques et pragmatiques sur le rapport entre les participants: Sujet, Objet et leurs Compléments. On analyse des exemples de deux corpus de registre oral: Le Corpus Sociolinguistique de la Ville de Mexico et Le Corpus du Parler de Monterrey. On montre la tendance suivante: les objets aliénables apparaissent avec des compléments prédicatifs, pas ainsi avec des objets inaliénables, où la structure préférée se combine avec des compléments adverbiaux ou prédicatifs secondaire qui habilitent et, dans quelques cas, renforcent la situation discursive.

Mots clés: apporter étatif, événement imperfectif, complément prédicatif, complément adverbial, prédicatifs secondaires, aliénables-inaliénable, espagnol de Mexico.

\section{Trazer estativo no espanhol do México: sua estrutura sintática e propriedades semânticas}

\section{Resumo}

Esta pesquisa teve como objetivo descrever os usos do verbo 'trazer' em eventos imperfectivos no espanhol do México, com o apoio na Gramática de Construções de Goldberg (1995) e em um estudo prévio sobre o 'trazer' estativo, de Rábago e Alarcón, 2012. Exploram-se algumas aristas sintáticas, semânticas e pragmáticas acerca da relação entre os participantes: Sujeito, Objeto e seus Complementos. Analisam-se exemplos de dois corpora de registro oral: 0 Corpus Sociolinguístico da Cidade do México y 0 Corpus da Fala de Monterrey. Mostra-se a seguinte tendência: os objetos alienáveis aparecem com complementos predicativos, não assim com objetos inalienáveis, onde a estrutura preferida se combina com complementos adverbiais ou predicados secundários que habilitam e, em alguns casos, reforçam a situação discursiva.

Palavras chave: trazer estativo, evento imperfectivo, complemento predicativo, complemento adverbial, predicados secundários, alienável-inalienável, espanhol do México. 


\section{Introducción}

La definición básica del verbo 'traer' remite al movimiento de un objeto, generalmente de un lugar al sitio donde se habla; 'traer' es un verbo que implica el traslado de un objeto, prototípicamente concreto, y la proximidad de este con el que habla, ejemplos (1) ${ }^{1}$ :

a). "I" Y allá me / tocó ver una cosa... / unas tortugas

"I"> Que le 'icen huachitonte / algo así / hast' eso me traje un caparazón de tortuga de allá de'sas

(MONR H11 HMP002) ${ }^{2}$

b). "E" >iNo tienes frío así como andas?

"I"> Sí

"E">A ver

"I"> No / ahi traigo suéteres

(MONR H11 HMP001)

Desde el punto de vista sintáctico-semántico, (1a) representa el uso básico de 'traer' con un sujeto agente, humano y capaz de transportar un objeto concreto, caparazón, de un lugar (fuente) de "allá", a otro (meta) que coincide con el lugar donde se encuentran el informante "I" y el entrevistador "E". En cambio, (1b) muestra la relación de proximidad entre el sujeto y el objeto, en este caso un objeto de su dominio personal ${ }^{3}$ (ahí traigo suéteres.)

1 Ejemplos tomados del Corpus del Habla de la Ciudad de México (CSCM) y del Corpus del Habla de Monterrey (HdeM). Las iniciales "I" se refieren al informante, y las iniciales "E" al entrevistador.

2 Al final de cada ejemplo se incluye la referencia completa de la fuente, MONR H11HMP002 corresponde al corpus HdeM y ME-268-22M-06 corresponde al CSCM. Estas etiquetas indican el número de la entrevista, Género, Edad, Educación, Trabajo Migración, Colonia y Salario y se mencionan para facilitar su consulta.

3 El concepto de dominio personal incluye objetos y seres asociados con una persona en forma habitual, íntima u orgánica: el cuerpo, sus partes, ropa, familia, etc. Cada uno se concibe no como una entidad simple, sino como una parte integral de la persona (Bally, C. 1996, p. 33). 
Las características principales del verbo 'traer' remiten al cambio de locación de un objeto, prototípicamente concreto, al lugar en o del que se habla (DRAE, 1992); el diccionario Espasa Calpe (1998) coincide con esta definición, pero añade la idea de proximidad: "transportar una cosa al sitio en que está el que habla, llevar puesto o consigo un objeto de la esfera personal". En la mayoría de las acepciones, el uso prototípico involucra el movimiento de un objeto, normalmente concreto, en un eje espacial como se puede observar en la figura 1 p. 4, donde se ilustra el sentido de trayectoria de X, desde algún lugar hasta el punto de llegada. En (1a), me traje un caparazón de tortuga de allá, se muestra la trayectoria recorrida hacia el lugar donde se ubica el hablante, este fenómeno coincide con el de la figura 1 :

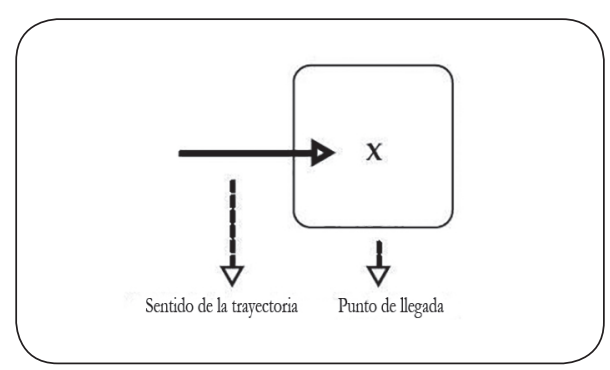

Figura 1. (Tomada de Pereira, 2013, p. 142)

Sin embargo, el verbo traer también aparece en contextos donde el movimiento de un objeto, siendo esta la característica principal de su uso, deja de ser evidente como se puede apreciar en (2):

2) "I": y ya se hacen los tomados < toma:dos>/ y que "¿sabes qué?/ no traigo dinero"/ "óyeme/ pero/ entonces < entós>/ estás consumiendo desde a qué horas y cómo/a la hora de pagar/ ya no traes dinero"/ ¿̇no? [ME-049-21H-99]

En (2), a diferencia de (1), no hay alusión a un movimiento4. La oración muestra la posición del objeto en el dominio del sujeto por un tiempo no especificado. Este evento deja fuera de la escena el punto inicial y el punto final de traslado. Nótese pues que 'traer' se puede interpretar de manera muy parecida a como lo hace el verbo 'tener'; inclusive, en (2), 'traer' podría sustituirse por 'tener' como en (3):

4 (Estás consumiendo desde hace horas), el contexto permite suponer que la persona no se encuentra trasladándose de un lugar a otro, se reitera el hecho de que el sujeto ha permanecido en el mismo lugar por algunas horas. 
3) "I" Estás consumiendo desde a qué horas y cómo/ a la hora de pagar/ ya no tienes dinero/ ¿no?

Este uso de 'traer', como se ilustra en la figura 2, deja fuera de foco los puntos iniciales y finales de la trayectoria así como el punto de llegada del evento y solamente se enfatiza la situación o estado en el que se encuentra X en el momento del discurso.

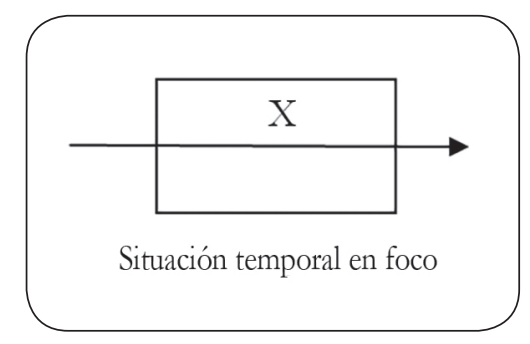

Figura 2. (Adaptada de Pereira, 2013)

Por consiguiente, el objetivo de esta investigación es analizar los usos de 'traer' en contextos que colindan con el verbo 'tener'. En esencia, se describe el uso de 'traer' donde ya no se refiere a un traslado en el eje espacial, sino que alude a una situación temporal de carácter estativo. Considera la estructura sintáctica de la oración; la duración del evento; así como algunos rasgos semánticos de los participantes en: sujeto 'humano', 'no humano' y ‘situación'; así como la clasificación de los participantes en: objeto 'concreto', 'abstracto', 'sensación física o psicológica', y 'parte del cuerpo'. Desde el punto de vista aspectual, la característica principal de 'traer' que aquí se plantea, se refiere a eventos imperfectivos y predica sobre el estado actual enmarcado en un evento temporal del sujeto y del objeto.

'Traer' en su uso básico como verbo de traslado, se clasifica como un verbo de logro5, el ejemplo en (1a) me traje un caparazón de tortuga de allá, se puede interpretar como un evento perfectivo (véase figura 1). Dicho evento coincide, según Givón (1984), con un evento focalizado en su totalidad como se esquematiza en la figura 3:

5 Vendler, Z. (1957, p. 146) clasifica los verbos en: estado, realización, logro y actividad. Esta clasificación ha sido de gran utilidad para entender la aplicabilidad de los verbos dependiendo de sus propiedades inherentes. 


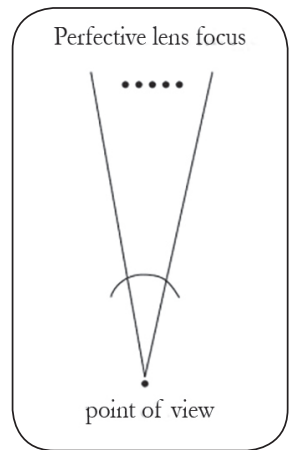

Figura 3 (Tomada de Givón, 1984, p. 289)

Cuando el acaecimiento con 'traer' se mira a través de una lente imperfectiva, se refiere a un evento inconcluso, donde quedan fuera de foco los elementos de inicio y fin, y permite ver solo una parte específica del evento.

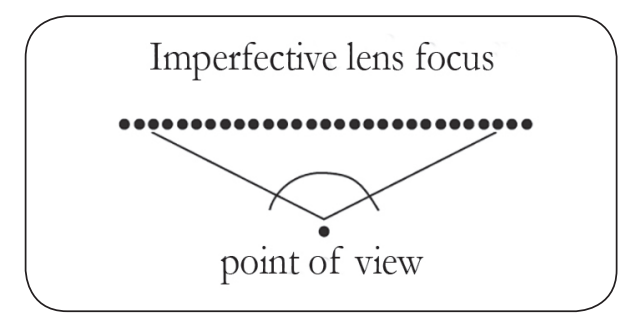

Figura 4 (Tomada de Givón, 1984, p. 289)

La figura cuatro muestra la descripción de un evento con una visión imperfectiva, en ella se omiten los puntos iniciales y finales del traslado en el eje espacial (fuente y meta), lo cual permite que el sentido de la oración pueda variar y se pueda interpretar como 'alguien' tiene 'algo' en su dominio: el ejemplo en (2), estás consumiendo desde a qué horas y cómo/ a la hora de pagar/ ya no traes dinero"/ ¿no?, permite interpretar la escena de una manera similar si utilizara el verbo 'tener': cómo/ a la bora de pagar/ ya no 'tienes' dinero"/ ¿no? En este ejemplo se suprime la idea de traslado de este, y 'traer' puede entonces ser interpretado como un verbo estativo (Rábago y Melis, 2005). 
El uso de 'traer' en un evento imperfectivo permite suponer que el movimiento ya no es evidente ${ }^{6}$, aunque el verbo muestra las siguientes características:

a. La proximidad de dos participantes (sujeto y objeto).

b. El evento se enmarca en un tiempo específico y deja fuera de foco la fuente y la meta.

c. Se sustituye el cambio de locación por una situación temporal, o estado en el que se encuentra el objeto enmarcado en el momento del habla.

Givón (1984) explica que el movimiento lineal pasa a conceptualizarse de forma virtual, de manera que se mantiene la atención durante el tiempo que permanezca el objeto en cierto estado. Esta característica aplica generalmente para verbos de movimiento. Visto así, 'traer' mantiene su rasgo original de desplazamiento, pero ya no en un eje espacial, sino en un eje temporal, por lo que comúnmente aparece en usos relacionales de tres tipos: de posesión, yo traigo un taxi; de atribución, hay unas telas que traen unos puntitos; y de afectación, traía el brazo enyesado (Rábago y Alarcón, 2012). En contextos donde 'traer' aparece como verbo de estado con una duración relativamente corta ${ }^{\top}$ coincide, según la clasificación de Givón (1984), con los verbos estativos temporales, el evento temporal alude a 'sensaciones físicas o psicológicas'; como el frío, el enojo, el hambre, o también a estados en los que se encuentran los participantes sujeto y objeto que lo acompañan, como se ilustra en este trabajo.

A continuación se describe la metodología utilizada para llevar a cabo este estudio en el cual se muestran ejemplos de distintas construcciones con uno o más participantes. La sección tres puntualiza los distintos tipos de construcciones y objetos analizados con

6 Langacker (1987) dice que el movimiento hipotético de un objeto concreto puede ser recorrido de manera mental por el hablante.

7 Retomando la clasificación de los verbos de Vendler (Z. 1957, p. 144) e integrando la propuesta de Givón (1984, p. 288) que incluye una sub clasificación más detallada de los tipos de verbos de acuerdo con sus propiedades inherentes, es preciso conocer su clasificación en 4 categorías: verbos compactos, de logro, de actividad y de estado. Los verbos compactos tratan de un evento perfectivo, como escupir, parpadear, toser. Los verbos de logro se caracterizan por enfocarse en la parte final del evento, como terminar, obtener; llegar. Los verbos de actividad se refieren a actividades o procesos que son susceptibles de visualizarse como una acción específica en un momento determinado.

Estos verbos a su vez se dividen en dos categorías Givón (1984, p. 288), los de corta duración o shorter son aquellos en los que a pesar de tener una estructura completa del evento, dejan fuera de foco la duración del evento desde una perspectiva comunicativa. Los verbos de actividad se dividen en verbos de mayor duración que el autor denomina longer como trabajar o leer, que al igual que los anteriores son susceptibles de dejar fuera de foco los puntos inicial y final, a menos que se requieran aspectos gramaticales que especifiquen la duración. Finalmente, los verbos de estado describen un evento de larga duración cuyos puntos iniciales y finales no se encuentran en escena.

Los usos del verbo traer que aquí se analizan corresponden, según Givón (1984, p. 288), a los verbos estativos temporales. 
'traer' no prototípico. La sección cuatro contiene el análisis cuantitativo de los datos. Por último, se presentan algunas conclusiones y recomendaciones para investigaciones futuras.

\section{Metodología}

Esta investigación es un diseño mixto que considera los datos de forma cuantitativa y analiza los contenidos de manera cualitativa siguiendo a Goldberg. Datos de dos corpus integrados por entrevistas procesados por medio de WordSmith.

Se analizaron 343 construcciones de El Corpus del Habla de Monterrey y Corpus Sociolinguístico de la Ciudad de México cada uno integrado por 108 entrevistas que se realizaron de manera oral. Se identificaron todos los usos de 'traer' que aquí se analizaron. No se consideraron los eventos enmarcados con aspecto perfectivo, 'traer' aparece conjugado solamente en tiempos presente y copretérito. Tampoco se han considerado usos de perífrasis verbales, ni usos auxiliares ni metafóricos. Las estructuras encontradas se caracterizan por tener un participante sujeto, sin ningún otro participante: traer + objeto. Aquí hay que mencionar que a pesar de que se encontraron estructuras $\mathrm{S}+\mathrm{V}+\mathrm{O}+\mathrm{MAtr}{ }^{8}$, estas no forman parte del análisis, debido a que solamente modifican al objeto al que acompañan y no al evento; no obstante, son frecuentes en los córpora.

Se organizaron los ejemplos en una base de datos de Excel. Cada uno de los ejemplos se clasificó de acuerdo con su estructura sintáctica y las propiedades semánticas de los participantes. Las clasificaciones son: objetos concretos, abstractos, sensaciones físicas 0 psicológicas, o partes del cuerpo.

En las construcciones analizadas, 196 ejemplos aparecen con la estructura $\mathrm{S}+0$, con el sujeto implícito y sin ningún modificador (cf. (1b)).

En el segundo grupo de construcciones aparecen con MAtr 56 ejemplos, $\mathrm{S}+\mathrm{V}+\mathrm{O}+\mathrm{MAtr}(\mathrm{cf} .(2 \mathrm{a}))$.

El tercer grupo analizado contiene la estructura $\mathrm{S}+\mathrm{V}+\mathrm{O}+\mathrm{CAdv}$, con un total 54 (cf. (2c)).

El cuarto grupo muestra la estructura de $\mathrm{S}+\mathrm{V}+\mathrm{O}+\mathrm{CPred}$ con un total de 15 construcciones (cf. (2b)).

8 S=Sujeto, O=Objeto, V=Verbo, CC=Complemento Circunstancial, CPred=Complemento Predicativo, PSec=Predicado Secundario, MAtr=Modificador Atributivo, CAdv=Complemento Adverbial. 
Y finalmente, los usos de la predicación secundaria con el verbo 'traer' muestran un total de 22 construcciones $\mathrm{S}+\mathrm{V}+\mathrm{O}+\mathrm{PSec}$ (cf. (2d))

\section{Tipos de construcciones en que se realizan los usos de 'traer' no prototípicos (estativos temporales)}

Las construcciones que se identificaron con el uso de 'traer' estativo difieren sintácticamente de aquellas donde el uso del verbo no varía. Estas construcciones se componen de un sujeto, un objeto, y otros participantes que cumplen con algunas funciones que en algunos casos modifican a uno o más participantes; en otras, funcionan como complementos de la oración.

De acuerdo con Sánchez (1999), Dowty (2000) y Aissen (2006), los complementos y los adjuntos (incluidos los predicados secundarios) se caracterizan por la función que desempeñan en la estructura oracional. Las diferencias esenciales entre los complementos y adjuntos se pueden mostrar tanto en el plano semántico como en el plano sintáctico (Dowty, 2000). Véase la tabla (1):

\begin{tabular}{|c|c|c|}
\hline & Complemento & Adjunto \\
\hline Sintácticamente & $\begin{array}{c}\text { Un complemento es un elemento } \\
\text { obligatorio }\end{array}$ & $\begin{array}{c}\text { Un adjunto es un elemento } \\
\text { opcional }\end{array}$ \\
\hline Semánticamente & $\begin{array}{c}\text { Un complemento completa el } \\
\text { significado del núcleo }\end{array}$ & $\begin{array}{c}\text { Un adjunto modifica el } \\
\text { significado del núcleo }\end{array}$ \\
\hline
\end{tabular}

Tabla 1(adaptada de Dowty, 2000)

En la tabla 1 se puede apreciar que los complementos son elementos sintácticamente obligatorios y semánticamente completan el significado de la oración dándole sentido. Por el contrario, los adjuntos son elementos sintácticamente opcionales en la oración, y cumplen con la función de modificar el significado del participante. La obligatoriedad/ opcionalidad de los participantes es indispensable para distinguir entre los tipos de construcciones que aquí se analizan. Los complementos predicativos (CPred) forman parte del predicado; por lo tanto, son obligatorios (Schultze-Berndt y Himmelmann, 2004). En (4a) morado se clasifica como CPred del objeto ojo, debido a que es obligatorio, porque completa información acerca del estado en que se encuentra la parte del cuerpo. Visto así, la ausencia de dicho CPred formaría una construcción incompleta como en (4b):

4)

a) "I" yo no sabía/ al día siguiente de que nos habíamos peleado/ yo ese día fui con mi mamá a la merced mercés/ y ya en la tarde él vino/ llegando de 
trabajar/ como traía el ojo morado y todo/ vino y le dijo a mi mamá dice/ "mire lo que me hizo su hija"

[ME-268-22M-06]

b) "él vino llegando de trabajar como traía el ojo / vino y le dijo a mi mamá dice/ "mire lo que me hizo su hija"

A diferencia de los CPred, los PSec son aquellos que modifican simultáneamente al verbo y a un sintagma nominal en la oración (Demonte y Masullo, 1999, Schultze-Berndt, et al. 2004, p. 77). Este tipo de predicación está relacionado funcionalmente con los modificadores atributivos, en cuanto a que ambos reflejan una eventualidad relacionada con el participante del predicado principal, y aluden a una función temporal que describe el estado en que se encuentra el participante simultáneamente al momento del evento del predicado primario. Los predicados secundarios se caracterizan por ser sintácticamente susceptibles a omitirse sin afectar la estructura gramatical entre los constituyentes principales (Schultze-Berndt et al., 2004). Esta característica los ubica como adjuntos dada su naturaleza de ser opcionales, véase los ejemplos en (5):

5)

a) "I">Que traigo gente capacitada / sí traigo gente capacitada / pero traigo un'o dos que me anda'ciendo por ahí grilla / pos ahí... / me les voy a pegar / y voy a hace'los que trabajen / al mismo / nivel que los otros [MONR H32 HMP088]

b) sí traigo gente / pero traigo un'o dos que me anda'ciendo por ahí grilla / pos ahí... / me les voy a pegar / y voy a hace'los que trabajen / al mismo / nivel que los otros

El modificador capacitada que aparece en (5a) cumple con los criterios de Palancar y Alarcón (2007, p. 338) para ser considerado como un predicado secundario, ya que se relaciona de manera temporal con el predicado principal y es susceptible de omitirse sin afectar la estructura gramatical de la oración como en (5b). ${ }^{9}$ En los casos donde la ambigüedad de las construcciones es evidente, la función pragmática de los adjuntos que se infiere del contexto puede ser una herramienta de análisis idóneo para establecer la necesidad de la participación de algunos modificadores en la construcción. Recuérdese que la función principal de la pragmática es la de satisfacer el requerimiento focal de

9 Palancar y Alarcón (2007, p. 338) mencionan que Halliday (1967) y Napoli (1989) consideran las construcciones 'George left angry' y George left angryly como del mismo tipo. Y añaden que de acuerdo con Plank (1985) y Winkler (199, pp. 325331 ), tanto predicados secundarios como complementos predicativos transmiten predicaciones parcialmente independientes acerca del participante del predicado principal. Este trabajo considera las principales diferencias entre predicados secundarios y complementos predicativos de Schultze-Berndt y Himmelmann (2004, p. 77). 
nueva información en la construcción. La existencia de adjuntos "obligatorios" en la modificación y en la predicación puede ser justificada desde un punto de vista pragmáticoconversacional, más que desde el punto de vista estructural. No es de sorprender que la obligatoriedad de algunos adjuntos, tanto en predicación como en modificación, se entienda primordialmente desde la pragmática discursiva general. Desde esta perspectiva, el requisito de que toda expresión tiene un foco que sirve para transmitir nueva información se cumple a través del uso de adjuntos "obligatorios". El acto comunicativo desde un punto de vista pragmático, se justifica bajo la propuesta de que la predicación o la modificación de un argumento son permitidas únicamente cuando este es relevante en el contexto del discurso (Goldberg et al, 2001) como puede apreciarse en (6):

6)

a) "I": yo ando trabajando / en el pinche taxi...Sí / y limpio / a mi me da gusto / l'igo a poco no / a mi me da mucho gusto que m'igan / oiga qué limpio trae su carro lo felicito / jah! gracias / a mí me gusta traerlo limpio

[MONR H21 HMP040]

b) a mí me gusta traerlo e?

En (6b) se puede apreciar que la omisión del PSec limpio, a pesar de ser opcional, es necesario para complementar la información dada en la construcción, de otra manera, se podría pensar que el informante expresa que le gustaría traerlo en cualquier otra condición o estado, sucio, con placas, con permiso, con las luces encendidas, etc.

En Rábago y Alarcón (2012) se mostró estadísticamente que existe una relación de dependencia entre el tipo de objetos poseídos (concretos, abstractos, sensaciones físicas o psicológicas, y partes del cuerpo) y las construcciones en las que aparecen en los casos analizados. En principio, debido a que 'traer' algo, en su acepción prototípica, predica el traslado de un objeto concreto de un lugar a otro, remite a la idea de que lo que se traslada puede ser controlado y manipulado por el sujeto. Cuando el verbo se muestra en una construcción estativa, su función se limita a predicar una relación entre dos participantes, sujeto y objeto, tal como lo haría el verbo 'tener'. Debido a esta semejanza, no es de sorprender que los objetos con 'traer' se encuentren en el dominio del hablante y se puedan considerar más o menos alienable ${ }^{10}$ tomando en cuenta sus propiedades semánticas como se muestra en la figura 5:

10 Se denomina posesión inalienable porque el objeto poseído no se puede enajenar. Los sustantivos que normalmente aparecen en esta categoría son partes del cuerpo, ciertas facultades y capacidades, así como objetos de la esfera personal. Nueva Gramática de la lengua Española: Manual (2010). Es importante notar que con verbos que no son considerados como posesivos, como el caso de 'traer', se difumina la interpretación de inalienabilidad. Lo que permite suponer que existe una línea difusa entre inalienable 0 alienable a la hora de clasificarlos. 


\begin{tabular}{|lcc|}
\hline POSSESSIVE SUBTYPE & PERMANENT CONTACT & CONTROL \\
Alienable & + & + \\
Inalienable & + & - \\
Temporary & - & + \\
Abstract & - & - \\
\hline
\end{tabular}

Figura 5. (Tomada de Stassen, 2009, p. 17)

Algunos de los objetos encontrados en las construcciones analizadas son inalienables al sujeto, como las partes del cuerpo; estas se clasifican como inalienables, ya que cumplen con los rasgos de [+contacto permanente] y [-control] (véase la figura 5). Las sensaciones físicas o psicológicas, las preocupaciones, estados de ánimo y experiencias físicas coinciden con la clasificación de temporales [-permanentes] y $[+$ control]. Finalmente, los objetos del tipo abstracto, como las ideas se caracterizan por ser [-permanentes] y [-control]. Como depende de los rasgos que caracterizan a los objetos de la esfera personal, resulta difícil delimitar su propiedad de alienabilidad con el sujeto. Debido a lo anterior, se encuentra una línea difusa entre alienable e inalienable y cabe mencionar que algunos autores se refieren a esta propiedad como inherente (Bally, 1996), en tanto que otros argumentan a favor de la propiedad no inherente del objeto en relación con el sujeto (Chappell y McGregor, 1996; Velázquez-Castillo, 2000; Seiler, 2001; Stassen, 2009). Los objetos concretos y objetos humanos pueden ser considerados como no inherentes ${ }^{11}$ al sujeto con el que aparecen. Esta propiedad de inherencia genera cierta ambigüedad a la hora de clasificar los CPred, los CAdv, los PSec, y es aquí donde el contexto es de gran utilidad para determinar su participación, obligatoria u opcional, en la construcción.

\section{$2.1 \mathrm{~S}+\mathrm{V}+\mathrm{O}$}

La mayoría de los casos encontrados en los córpora se limitan a predicar una relación entre el sujeto y el objeto, sin ningún tipo de participante que modifique o complemente la oración como en (7a, b y c).

7)

a) "I": y sí es así como que/ y yo dije/ no/ ya// y ahora la maestra de jardín es la que lleva la/ tiene una como que/ tiendita// entonces < tons $>$ a la hora que

11 Sujetos humanos no inherentes se consideran las relaciones que excluyen parentesco (padres, hijos, hermanos, nietos, etc.), las relaciones de amistad, en cambio, se conocen por tener una duración limitada y que puede ser controlada por el sujeto (por control nos referimos a que el sujeto tiene la opción de continuar con la relación o de terminarla en el momento que lo desee). 
ella la pone/ yo estoy ahí en su salón/ entonces dice/ "a ver/ ¿qué compraste ahora C?/ ay/ traes chocolates/ ay traes galletitas/ ay yo quie-" (risas)

[ME-214-21M-02]

b) "E": Y ahora estás en en un pedo de de que mejor trabajas / y juntas feria para... otro pedo

I: Sí / no / pos orita haz de cuenta / orita... / me ando calmando porque... / pu's / ya traigo una morrita ¿veá? ya traigo una novia y... / y pu's

[MONR H11 HMP005]

c) "I": Es normal / es como si yo fuera al DF y les dijera ¿sabes qué?'ste / traigo una chaqueta porque'ste / traigo frío / y allá chaqueta es otra cosa que aquí en Monterrey es muy distinta dices tú jah canijo! (risa)

[MONR H12 HMP017]

Como se puede ver en los ejemplos, en ( $7 \mathrm{a})$, se hace referencia a objetos que tienen ciertas características, los tipos de objetos que aparecen con 'traer' pueden ser concretos chocolates, galletitas; humanos (7b) una novia, y experiencias físicas o psicológicas como en (7c) frío. En todos los casos, aparecen sin otro participante modificando al objeto.

\subsubsection{S+V+O+ Modificador atributivo}

Otro tipo de construcciones con 'traer' se caracteriza por aparecer con un sujeto (implícito en este caso) acompañado del objeto sangre y un modificador nominal, coabuilense, que solamente modifica al objeto ${ }^{12}(8 \mathrm{a})$ :

8)

a) "I" Y allá me / tocó ver una cosa... / unas tortugas

"I"> Que le 'icen huachitonte / algo así / hast' eso me traje un caparazón de tortuga de allá de'sas

(MONR H11 HMP002)

b) "E"> $>$ No tienes frío así como andas?

"I"> Sí

"E">A ver

12 En este ejemplo, por referirse a una atribución gentilicia del participante sujeto, el MAtr es requerido. En otros casos, este MAtr puede no estar presente como en traía un vestido de holanes vs. traía un vestido. 
"I"> No / ahi traigo suéteres

(MONR H11 HMP001)

8) a. I: Yo crecí / crecí en Reynosa Tamaulipas / nací en Poza Rica Veracruz / pero... / la sangre... / que traemos es / por parte de mi padre / de Coahuila / por parte de mi padre traigo sangre coahuilense y por parte de mi madre que nació en Cerralvo Nuevo León / precisamente / traemos el Ramos Salinas [MONR H23 HMP061]

\subsection{S+V+0+CPred}

También aparecen construcciones con objetos acompañados de complementos predicativos, sintácticamente obligatorios, véase los ejemplos en (9) donde el objeto, al ser una parte del cuerpo, exige la presencia del cPred para que toda la construcción cobre sentido y no se muestre como una construcción sin un contexto que permita mostrar una independencia sintáctica *traigo la mano.

9)

a) "I": Este... / simplemente mira / yo traía la mano / volteada al revés así E: Ajá / como... / entumida: No / de’ste to'avía 'tá entumida la mano / pero ya la puedo flexionar y hacer / hacer ejercicios / pero yo la traía'sí mira [MONR H32 HMP088]

b) "I”:[sí/ sí]/ ¿quién sabe de...?/ yo creo que de la tierra salió un/ un policía [que] nunca están ahí I:de repente salió y/ "a ver/ y esto/ y lo otro/ y"/ yo traía/ bueno/ estaba asegurado el carro de ahí/ yo traía mis papeles bien/ el otro chavo tra-/ no traía licencia

[ME-284-12H-07]

En (9.b) se tiene un objeto de la esfera personal, que al igual que las partes del cuerpo, se encuentra dentro de la jerarquía de rasgos semánticos en el extremo de lo inherente. Por ello, el CPred es exigido en la construcción.

\subsection{S+V+O+CAdv}

La estructura sintáctica caracterizada por el verbo 'traer' aparece con complementos adverbiales funcionando como circunstanciales. Estos complementos refuerzan la idea de que 'traer' estativo se especializa en eventos temporales, válidos para el momento del discurso, donde el sujeto y el objeto se encuentran en un mismo espacio, se predica sobre eventos imperfectivos en los que es necesario, a través de los CC, detallar la información del evento con mecanismos sintácticos como se puede ver en (10): 
10) "I": muchos compatriotas llegaron a matarlos por robarles su dinero/ hay mucha este/ hay mucha este/ ¿cómo se puede decir?// pues < pus $>$ se ve un/ se mueve mucho la droga/ lo que es en barrio de/ de negros/ mueven mucho la droga y/ y te digo/ asaltan al/ al hispano/ saben que el hispano/ el fin de semana trae dinero en la bolsa de doscientos trescientos/ de doscientos dólares para arriba/ traen dinero en la bolsa/ y no falta/ que los/ se los encuentren/ los atacan/ les quitan su dinero/y a varios los llegaron a matar [ME-275-22H-06]

El ejemplo en (10) muestra que se requiere de un CC 'en la bolsa', obligatorio desde el punto de vista de Goldberg (2001, p. 805), "without any focal information clausal predication is generally infelicious", tomando esto como referencia, para que la oración pueda cumplir con la información específica que el hablante intenta comunicar; el contexto en el que se emite la construcción demuestra la importancia que cobran algunos elementos sintácticos para reforzar propiedades pragmático-discursivas de la lengua por encima de clasificaciones gramaticales (Goldberg et al., 2001).

\section{$2.4 \mathrm{~S}+\mathrm{V}+\mathrm{O}+\mathrm{PSec}$}

El último tipo de construcciones que aparece con 'traer' se relaciona con el uso de predicados secundarios. Véase los ejemplos en (11):

\section{1)}

a) "I": Le dije ya lo v'a 'star esperando ahí / yo me voy a regresar l'o luego no me voy a tardar / bueno / no de ahí se lo llevaron al 25 E: Sí I: De volada / de la 2 / porque traía un infarto enmascarado / dijeron E: ¡Ah! ¡isí? / ¿cómo / cómo es eso?: Pos que parece infarto y que no parecen / quién s'e qué E: ¡Válgame! / fíjese

[MONR M32 HMP092]

b) "E": Luego el cigarro apesta

"I": Sí E: Se apestan los autos. Sí sí / siempre / siempre traigo el aromatizante ahí / se suben al carro y dicen / oye qué bonito huele / gracias / trae muy limpio su carro jah! muchas gracias [MONR H21 HMP040]

En (11a), la estructura se compone de un predicado primario 'traía un infarto', y de un predicado secundario ‘enmascarado’ que modifica simultáneamente al verbo principal traía y al objeto 'infarto' en la construcción; una situación similar sucede en (11b), el predicado primario ‘trae su carro', y el predicado secundario 'muy limpio' en el que existe 
una modificación simultánea entre el sujeto y el verbo de la predicación principal 'trae'. La opcionalidad del PSec se ratifica en el hecho de que pueden existir las construcciones de (12) sin que se cuestione su estructura sintáctica plena $(\mathrm{S})+\mathrm{V}+\mathrm{O}$ :

12)
a) (alguien) (S) traía $\mathrm{V}$ un infarto 0 .
b) (alguien) (S) trae $\mathrm{V}$ su carro 0.

\subsection{Tipos de objetos con 'traer' no prototípico}

Las propiedades de los objetos que acompañan al verbo 'traer' en usos estativos juegan un papel crucial para entender lo que ocurre con las construcciones. Cuando los objetos son totalmente inherentes al sujeto, como las partes del cuerpo, la estructura sintáctica involucra la participación de un CPred (obligatorio) para describir el estado en que se encuentran los objetos 'mano' y 'presión'; véase el ejemplo en (13):

\section{3)}

a) "I": Este... / simplemente mira / yo traía la mano / volteada al revés así No / de'ste to'avía 'tá entumida la mano / pero ya la puedo flexionar y hacer / hacer ejercicios / pero yo la traía'sí mira

[MONR H32 HMP088]

b) "I":[bueno]/ mis actividades/ yo soy cirujano dentista/ si estoy aquí/ es por la sencilla razón/ de que este/ eh// me tomaron en cuenta/ la a nivel preventivo/ o sea/ medicina preventiva/ para dar aquí este// este/ pues/ diagnosticar a la gente// mm/ tomar su peso/ su talla:¿sí? darles también/ si si si traen la presión alta/ [presión baja]

[ME-139-32H-01]

En los usos estativos, los modificadores de objetos parcialmente inherentes, como los de la esfera personal, al no ser sintácticamente requeridos en la construcción, tienen la función de proveer información específica sobre el estado o la manera en que se encuentran los participantes del evento en el momento del discurso como se puede ver en (14):

14) "I" pero así nomás agarra y se volteaba a ver/ y volteaba a ver/ dije "este cuate"/ y ya ves que siempre traigo pantalones así/ ajustados (risa)

[ME-291-11H-06] 
En (14), los pantalones, objeto de la esfera personal del hablante, están modificados por el adjetivo ajustados, que funciona como un PSec; destaca como usa la prenda el taxista. Otro de los tipos de sustantivos encontrados se refiere a objetos abstractos como en (15) pensamiento, ideas, que habilita el uso con la estructura $S+V+0$.

15)

a) "E": sí me cae < cai> que sí está rudo// sí yo creo que deberían/ pues $<\sim$ pus > cambiar a fondo ¿no?/ todo lo/ lo rural

I: eso sí

"E": porque sí se las/ las llevan todas de perder mano ahí//

"I": [pues] a ver qué a ver qué pensamiento trae este

"E": [el Fox]

[ME-114-12H-00]

b) no hay tanta libertad por parte de la gente que tatúas/ ¿no?/ o sea/ muchas veces la gente viene y tú les sugieres lo mejor/ ¿no?/ claro/ porque tú/ a eso te dedicas/ ¿no?/ y sabes qué les convendría más a ellos/ pero bueno/ ellos traen un ya/ una idea/de lo que se quieren bacer//

[ME-103-21H-00]

Con objetos humanos, aparecen ejemplos que dependen, en gran medida, del tipo de relación que guardan con el sujeto. Se entiende que las relaciones de parentesco son inherentes, por lo tanto, podría considerarse un tanto ambiguo predicar sobre 'traer', en el sentido de 'tener' a un familiar, a menos que se especifique a través de un complemento, que este se encuentra en cierto estado, 'cargada', como en (16b), puede generar cierta ambigüedad a la hora de determinar los contextos en los que la modificación es opcional, esto depende del tipo de relación que se tenga con el objeto. Cuando se refiere a relaciones de parentesco.

16)

a) "I": Es muy difícil porque... / pensar que tu vida estuvo en un hilo / y más cuando traes en tu vientre una criatura $Y$ por eso le doy gracias a Dios de que / un día'ntes a mi me / me incapacitaron [MONR21 HMP045]

b) mi hermana pos creída ¿vedá? / traía' su niña de / ocho meses / cargada [MONR M22 HMP060]

c) "I": Sí / no / pos orita haz de cuenta / orita... / me ando calmando porque... / pu's / ya traigo una morrita ¡veá? ya traigo una novia [MONR H11 HMP005] 
En (16a), la criatura es hijo del sujeto, por lo tanto, se considera una relación de parentesco inherente al mismo. En (16b), de igual forma podemos suponer que su niña de ocho meses acompaña al sujeto, mas no se predica sobre la relación que existe entre ambos, ni tampoco se considera que dicha relación se terminará en algún momento por voluntad de los participantes. En cambio en (16c), la relación con un participante humano, 'morrita' ${ }^{13}$, predica que ambos tienen una relación amorosa que se puede terminar en cualquier momento. Aquí cobra importancia la relación que guardan los objetos humanos con el sujeto para poder decidir cuando son inherentes.

Los objetos concretos, que no guardan relación inherente con el sujeto, son susceptibles de separarse en cualquier momento de él. Tratándose de objetos que se encuentran en su dominio en el momento del discurso, se entiende que durante un tiempo específico, se mantendrán en contacto o en cercanía, pero dado su carácter de no inherentes, pueden separarse en cualquier momento como el sobre del aguinaldo y $\mathrm{el}$ chicle en (17).

a) "I" Yo traía el sobre del aguinaldo / entre mis libros ino? / y ya me 'icen a ver la mochila y me la quitan

[MONR H13 HMP029]

b) "I": una- unos be- unos baloncitos/ que tienen <una > madera/ aquí/ tiene pegado

"E": ah sí

"I": y ya ya son/ adentro trae el chicle

"I": de esos

"E": y los tienes que ir armando

"I": ajá sí nomás nos dan la pelotita y tenemos que meter el chicle/ el chicle nos lo dan contado

[ME-285-11M-07]

\section{Análisis cuantitativo de los datos}

Los datos analizados en los córpora vistos de forma esquematizada (véase tabla 2) permiten observar que el uso de las distintas construcciones descritas anteriormente depende en gran medida del tipo de objeto en la predicación, ya que 'traer' en función estativa describe un evento temporal en el que predominan: una relación entre dos

13 Término coloquial para referirse a una mujer joven que, en este contexto, la equipara a una novia. 
participantes S y 0 , el estado en el que se encuentran tanto el S como el 0 , y en algunos casos, la situación circunstancial que caracteriza el evento al momento del discurso.

\begin{tabular}{|c|c|c|c|c|c|}
\hline $\mathrm{S}+\mathrm{V}+0$ & $\mathrm{~S}+\mathrm{V}+0+\mathrm{MA}$ & $\mathrm{S}+\mathrm{V}+0+\mathrm{CPred}$ & $\mathrm{S}+\mathrm{V}+0+\mathrm{PSec}$ & $\mathrm{S}+\mathrm{V}+0+\mathrm{CAdv}$ & Total \\
\hline $196(57.14 \%)$ & $56(16.32 \%)$ & $15(4.37 \%)$ & $22(6.41 \%)$ & $54(15.74 \%)$ & $343(100 \%)$ \\
\hline
\end{tabular}

Tabla 2. Frecuencia de tipo de construcciones analizadas

La tabla 2 muestra la frecuencia con la que aparecen las diferentes construcciones con objeto solo, con complementos adverbiales, con complementos predicativos y con predicados secundarios. Nótese que el mayor número de ejemplos aparece con objeto solo, como traigo dinero. Esta tendencia estimula al hablante a ser más explícito utilizando algún tipo de modificador o complemento. En la tabla también se puede ver que el uso de construcciones con predicados secundarios (cf.(11a)) (6.41\%) es mayor al uso con complementos predicativos ( $c f$. (9a)) (4.37\%), es decir, la participación de modificadores o complementos en la oración es mayormente opcional, en tanto que la obligatoriedad de los participantes derivada de sus propiedades semánticas es menos frecuente.

La estructura $\mathrm{S}+\mathrm{V}+\mathrm{O}$, sin modificador, abarca en su mayoría objetos de tipo concreto, 'dinero', 'el sobre', 'fotografías', etc. Pero también involucra sensaciones físicas o psicológicas que de alguna manera expresan el estado actual en el que se encuentra el hablante. En las construcciones que muestran la estructura $\mathrm{S}+\mathrm{V}+\mathrm{O}+\mathrm{CPred}$, los CPred predican sobre las partes del cuerpo que son denotadas por los objetos 0. La predicación con este tipo de objetos no se refiere a la posesión de cierta parte del cuerpo, sino al estado en el que esta se encuentra. Considerando que la propiedad de inherencia de las partes del cuerpo no predica su posesión, sino su estado o condición temporal, se cree que esta propiedad relaciona los CPred con objetos del tipo 'partes del cuerpo', debido a la propiedad inherente del objeto (véase (11a)).

Para las construcciones con la estructura $\mathrm{S}+\mathrm{V}+\mathrm{O}+\mathrm{PSec}$, el uso de PSec está vinculado con objetos de la esfera personal, inalienables al sujeto, lo que supone la no obligatoriedad en la oración ( $c f$. (11)). Sin embargo, considerando que se trata de un evento temporal en el momento del habla, requiere la presencia del uso de un adjetivo en función de PSec para cumplir con la situación comunicativa. Esta línea difusa que impide diferenciar la gramaticalidad o aceptabilidad en la oración puede verse en (18). Cuando los elementos no son suficientes para hacer una interpretación adecuada de la construcción, entran en escena algunas consideraciones pragmáticas que permiten visualizar que la caracterización semántico-sintáctica no siempre es suficiente para darle sentido a las construcciones analizadas. Véase el ejemplo siguiente: 
18)

a) trae los tenis bien amarrados

b) trae los tenis

En (18a) se puede entender, desde el punto de vista sintáctico, como una cláusula gramatical $(\mathrm{S})+\mathrm{V}+\mathrm{O}+\mathrm{CPred}$. No obstante, analizada bajo la lente que aquí se propone, el ejemplo en (18b) es difuso. En principio, se trata de un referente específico, los tenis, no se habla de cualquier par de tenis, sino de un objeto que es parte del conocimiento mutuo entre los participantes en la escena. Visto así, la ausencia del modificador parece no afectar el significado principal de la construcción, se considera entonces una PSec 'amarrados'. Con un lente más fino, se requiere de un contexto que permita ubicar al interlocutor ante una situación discursiva donde se ponga en relieve la información nueva contenida en el PSec 'amarrados' de manera tal que se elimine ambigüedad con respecto a la posibilidad de suponer que el sujeto (implícito) en la oración traía los tenis puede también complementarse con un CAdv en la mano, en la mochila o con la agujetas sueltas.

Por último, en las construcciones que tienen la estructura $\mathrm{S}+\mathrm{V}+\mathrm{O}+\mathrm{CAdv}$, los $\mathrm{CAdv}$ aparecen con objetos de la esfera personal. Estos complementos refuerzan la idea de que 'traer' estativo se enfoca en una parte del evento temporal que tiene validez en el acto discursivo, donde el sujeto y el objeto se encuentran en un mismo espacio; en otras palabras, se predica sobre eventos imperfectivos en los que es necesario, a través de los CAdv, dar información adicional del evento a través de mecanismos sintácticos (véase el ejemplo en (10)).

En resumen, los sustantivos que aparecen en función de objeto en las construcciones analizadas son generalmente nombres que denotan partes del cuerpo 'cabeza', 'cara', 'mano', 'ojos', 'pies', lo que se ajusta de modo estricto a la posesión inalienable. Sin embargo, se consideran también sustantivos que designan objetos de la esfera personal 'vestimenta' 'camisa', 'pantalón', 'cinturón', 'casa', 'coche', 'paraguas', 'reloj', debido a que, para algunos autores (Stassen, 2009; Velázquez-Castillo, 2000; Seiler, 2001; Chappell, y McGregor, 2009) estos objetos presentan manifestaciones de posesión inalienable. De igual modo, se consideran como inalienables los sustantivos que denotan acciones corporales y sus efectos mirada, sonrisa así como ciertas facultades y capacidades 'amor', 'trabajo', 'planes' (Winters, 2009). Lo anterior se sustenta en el modo de concepción de las construcciones basado en aspectos pragmático-discursivos y su relevancia en la codificación-interpretación de información en el plano conversacional. 


\section{Conclusiones}

En este trabajo se han descrito algunos tipos de construcciones sintácticas y la relación que guardan con los objetos dependiendo de sus propiedades semánticas de los participantes en las construcciones con 'traer' en función estativa. La proporción del tipo de construcciones sintácticas ha corroborado que 'traer' puede aparecer en una construcción únicamente acompañado del objeto, véase (1b). Además, la composición sintáctica varía de acuerdo con las propiedades semánticas de los tipos de objetos que participan en el evento. Las propiedades de los objetos que acompañan al verbo 'traer' distinto al uso prototípico son de suma importancia para dar cuenta de lo que se está gestando en los eventos estativos. Cuando los objetos son inherentes al sujeto, como las partes del cuerpo, la estructura sintáctica más utilizada involucra la participación de un CPred (obligatorio) que lejos de cumplir con la función de predicar una relación entre el sujeto y el objeto, se dedica a describir el estado en que se encuentra el objeto.

Cuando se trata de objetos de la esfera personal, en el dominio del sujeto, 'traer' aparece más frecuentemente en construcciones con PSec. Cuando los objetos de la esfera personal no son inherentes al sujeto, la construcción sintáctica preferida involucra CPred, que a pesar de no ser obligatorios en la construcción, proveen información adicional sobre la manera en que se encuentra el objeto en el momento del discurso.

Nótese pues que una de las principales diferencias encontradas entre los tipos de construcciones en este análisis fue el rasgo sintáctico de obligatoriedad, pues juega un papel fundamental para la descripción de las construcciones aquí analizadas. No obstante, el punto de vista pragmático también cobra importancia cuando se describe el evento, no desde su formación sintáctica, sino desde el enfoque orientado a satisfacer la inclusión de información nueva obtenida con el modificador que acompaña al sujeto. Desde este punto de vista, el modificador puede o no adoptar el rasgo de obligatoriedad; ya no depende de la estructura sintáctica, sino de las propiedades semánticas del objeto y del contexto discursivo que determina la pertinencia del rasgo opcional u obligatorio con el fin de proveer la información nueva que se obtiene en la construcción; véase el ejemplo (14) que se repite aquí para conveniencia del autor:

14)

"I" pero así nomás agarra y se volteaba a ver/ y volteaba a ver/ dije "este cuate"/ y ya ves que siempre traigo pantalones así/ ajustados (risa) [ME-291-11H-06] 
Sintácticamente, 'ajustados' no es obligatorio, pero en el contexto donde el taxista explica que "este cuate" se volteaba a ver, de manera reiterativa a los pantalones del chofer, se debe precisamente a la forma en que llevaba puestos los pantalones, 'ajustados', característica que llamó la atención del pasajero. El rasgo de opcionalidad que subyace en los modificadores de los objetos en las construcciones con 'traer' en función estativa muestra, precisamente, que el uso estativo del verbo en cuestión se va en incremento en el habla cotidiana, y su uso está orientado a aparecer muy frecuentemente acompañado del objeto con la estructura $\mathrm{S}+\mathrm{V}+\mathrm{O}$ después aparecen los usos con la estructura $\mathrm{S}+\mathrm{V}+\mathrm{O}+\mathrm{CAdv}$. Cabe marcar que en la tercera posición en frecuencia tenemos $\mathrm{S}+\mathrm{V}+\mathrm{O}+\mathrm{PSec}$ seguido por los usos con CPred.

En este estudio, se abordaron cuestiones relacionadas con el uso de 'traer' estativo en el español de México, y se mostró que la predicación secundaria es utilizada por el hablante en el tipo de construcciones analizadas. Sin embargo, sería interesante llevar a cabo un estudio incluyendo otras variantes del español, con el fin de comprobar si existen acepciones del verbo 'traer' en su uso no prototípico que aquí se presentó. Finalmente, sería interesante hacer una revisión exhaustiva que permita detectar otros verbos que se comporten de manera semejante al fenómeno que aquí se describe.

\section{Referencias bibliográficas}

Aissen, J. (2006). “Taller sobre la Complementación”. México: Centro de Investigaciones y de Estudios Superiores en Antropología Social-Sureste.

Bally, C. (1996). The personal domain and the indivisibility in Indo-European Languages. En Chappell, H. y McGregor, W. (Eds.), The grammar of Inalienability. A Typological Perspective on Body Part Terms and Part-Whole Relation, pp. 31-61. Berlin: Mouton de Gruyter.

Chappell, H., \& McGregor, W. (1996). Prolegomena to a Theory of Inalienability. En The Grammar of Inalienability. A Typological Perspective on Body Part Terms and Part-Whole Relation, pp. 3-29. Berlin: Mouton de Gruyter.

Corpus Sociolingǘstico de la Ciudad de México. (2011) Materiales del PRESEEA-MÉXICO. Vol. I. Hablantes de nivel superior. Pedro Martín Butragueño y Yolanda Lastra. El Colegio de México.

Demonte, V., \& Masullo P. J. (1999). La predicación: los complementos predicativos. En: Bosque, I., \& Demonte, V. (Eds.) Gramática Descriptiva de la Lengua Española. Vol. II. pp. 24612523. Madrid: Espasa. 
Diccionario de la Lengua Española (1992). XXI Edición. Real Academia Española.

Dowty, D. (2000). The Dual Analysis of Adjuncts/Complements in categorial Grammar. ZAS Papers in Linguistics, 17, 53-78. Recuperado de http://www.ling.ohio-state.edu// dowty/papers/ degruyter.8x11.pdf

Givón, T. (1984). Syntax: a functional-typological introduction, Vol. I. Amsterdam: John Benjamins.

Goldberg, A. E., \& Farrell, A. (2001). The Pragmatics of Obligatory Adjuncts. Language, 77(4), 798-815.

Goldberg, A. E. (1995). Constructions: A construction grammar approach to argument structure. Chicago: The University of Chicago Press.

Langacker, R. W. (1987). Foundations of Cognitive Grammar. Standford: Stanford University Press.

Nuevo Diccionario Enciclopédico Espasa. Espasa Calpe S.A. (1998)

Palancar, E., \& Alarcón Neve, L. J. (2007). Predicación Secundaria Depictiva en Español. Revista Española de Lingüística (RSEL), 37, 337-370.

Pereira, D. I. (2013). Construcciones de movimiento causado con el verbo poner: un estudio de corpus. Literatura y lingüística, (27), 137-152. Recuperado de http://www.scielo. cl/scielo.php?script=sci_arttext\&pid=S0716-58112013000100008\&lng=es\&tlng=es. 10.4067/S0716-58112013000100008.

Rábago, Á., \& Alarcón, L. (2012). Diferencia entre depictivos y complementos predicativos que acompañan a los verbos traer y llevar en su uso estativo en el español de México. XII Encuentro Internacional de Lingüística en el Noroeste, pp. 1595-1600. Hermosillo: Universidad de Sonora.

Rábago, Á., \& Melis, C. (2005). El sentido posesivo de traer. Lingüística Mexicana, II(2), 227247.

Real Academia Española. Asociación de Academias Americanas, S.L.U. (2010) Nueva gramática de la lengua española; Manual: Espasa Libros.

Rodríguez, L. (2003). Base de información sobre El Habla de Monterrey. Monterrey: Universidad Autónoma de Nuevo León.

Sánchez Valencia, V. (1999). Semántica Aspectual y Predicación Secundaria. Crítica. Revista Hispanoamericana de Filosofía, XXXI (93), 49-74. 
Schultze-Berndt, E., \& Himmelmann, N. P. (2004). Depictive Secondary Predicates in Crosslinguitic Perspective. Linguistic Typology (8), 59-130.

Seiler, H. (2001). The operational basis of possession. A dimensional approach revisited. En Baron, M. Herslund \& F. Sorensen (Eds.), Dimensions of possession (27-40). Amsterdam: John Benjamins.

Stassen, L. (2009). Predicative possession. New York: Cambridge University Press.

Velázquez-Castillo, M. (2000). Posesión Inalienable en Español: Niveles de Tematicidad e Individualización (Vol. Monográfico). (R. Maldonado Soto, Ed.) México D.F.: Solar Servicios Editoriales.

Vendler, Z. (1957). Verbs and Times. Philosophical Review. Ithaca, LXVI(2) 143-160. N.Y.: Cornell University Press.

Winters, R. (2009). Expressing Possession in Spanish: The use of possessive datives and possessive adjetives in the Twentieth-Century Spanish Language Drama. Hispanic Research Journal, 10(4), 289-302. 